\title{
Digital video for the enterprise - A view from the trenches
}

\section{Tim Claxton}

is a senior product manager at Getty Images, where he is responsible for the development of services for storing, managing and sharing video, images and other multimedia assets. He is involved in all aspects of the development cycle, from roadmap planning, development to launch. Tim has $12+$ years of experience in technology and digital media. Prior to Getty Images, Tim was a senior product manager with Aventail Corporation, and served in several roles with IBM Global Services, including Principal, where he led a consulting team to help companies implement intranet, extranet and content management applications.

\section{Jon Folland}

is the director and co-founder of Nativ Ltd., a consulting, technology and outsourcing company specializing in the delivery of video-centric solutions. Before founding Nativ in 2001, Jon was a head of technology at a number of start-ups, managing the development of large-scale technical solutions for companies such as MTV and BBC. Prior to this, Jon was a technical team leader at Razorfish, UK, where he managed and mentored teams of developers in the delivery of technical solutions for high-profile clients such as British Aerospace, NMEC, Natwest and Britannia. Jon has over ten years' experience in management and IT.

Keywords: digital asset management, digital video, 360-degree distribution, softwareas-a-service, transcoding, streaming

Abstract In this paper, we detail some of the key technical challenges faced by asset managers in this emerging nonlinear world of digital video.

Journal of Digital Asset Management (2007) 3, 263-268. doi:10.1057/palgrave.dam.3650095

\section{INTRODUCTION}

An executive takes one look at YouTube, and before you know it, there is a mandate to implement digital video across the enterprise. This is quickly followed by "surely it cannot be that hard." Does this sound familiar?

Consumer video is changing the way that we feel about using digital video as a tool to promote our products and services - from Flash elements on a website to user-generated content or the latest $\mathrm{CEO}$ broadcast on $\mathrm{CNN}$ online.

Yet, the complexities are real, and moving to a tapeless world can require significant investment in technology and resources. And content often needs to be produced and made available to a growing number of platforms and devices, including the web and mobile. This emerging and exciting trend, which is increasingly being referred to as the "360degree" approach, introduces a new level of complexity and poses a steep learning curve. Loosely speaking, 360-degree distribution means delivering content to any platform or device, at any time. It is important to note, however, that it is entirely driven by customer convenience and personal choice.
So what does one need to consider when implementing video within the enterprise, in terms of technical and organizational bottlenecks, to deliver on the 360-degree promise? In this paper, we will discuss these challenges based on our experience from working with companies around the globe and also present Getty Images' Media Manager service as a potential solution to help you overcome them.

\section{CHALLENGE 1 - LEARN A NEW LANGUAGE}

The first challenge is the sheer proliferation of video standards. For instance, a QuickTime (MOV) file could use one of a variety of different video codecs, such as Sorenson 3 , PhotoJPEG, Motion JPEG a/b - the list goes on. There are also a variety of standards to meet for different uses across the web, mobile and broadcast. In addition, formats such as H264,VC-1, DivX and On2 are constantly evolving.

Many of the challenges with the 360-degree paradigm have not been met before, hence the continual announcements of new and overlapping standards. In fact, there are so many companies in 
the digital and rich media market working autonomously to define new "standards" from encoding and distributing to storing and protecting video that the word "standard" is becoming meaningless in this context. Vendors deviating from, or not adhering to, existing standards further compound the problem. It is all too easy for a vendor to stretch a specification in the interests of market edge and end-to-end domination, and at the expense of interoperability. One of the reasons this is happening is that strategic and technical aspects of the video marketplace are in a state of huge flux.

One way to overcome this challenge is to implement just one format that meets your needs or pick technologies that support a wide variety of standards.

\section{CHALLENGE 2 - SIZE MATTERS}

If you are used to working with images and PDF files that range from 5 to 10 megabytes, you will need to be able to accommodate significantly larger files for digital video formats. Let me outline some examples.

- One hour of a medium-resolution web clip requires about 250 megabytes.

- One hour of a standard-definition NTSC/PAL clip requires about 11 gigabytes.

- One hour of HD at $1080 \times 192024 \mathrm{PsF}$ requires about 670 gigabytes.

If you have not yet made the request for more storage on your network, you might want to consider it now.

\section{CHALLENGE 3 - SYSTEMS INTEGRATION}

As mentioned earlier, the simplicity of the "play button" does not indicate the technologies required to power your digital video. Here is a list of the key technologies you will need if you are thinking about implementing, building and integrating a video platform:

- Transcoding engine - to convert formats on upload or download.

- Storage - to handle the expected volume of files.

- Asset classification and metadata management - to organize assets and enable users to search/browse video assets in a library.
- Streaming - either streaming service or in-house servers to playout content to users.

- Delivery - network technologies for high-speed delivery of assets, especially for users outside the corporate network (ie remote offices, traveling, home DSL, etc).

From our experience, the melding of technologies with wider enterprise systems is still at an early stage and therefore specialist integration skills are required. Nativ's systems integration experience in the broadcast and digital video domain has shown that large product integrations can sometimes cost much more than the cost of the original product licenses - particularly when things go wrong. Consequently, the integration effort should not be underestimated or considered a trivial, cheap or low-risk task (regardless of what a vendor may promise!). Be cautious of buzzwords like "turnkey" and "out-of-the-box," and ask to see examples of successful integrations and the duration time to implement them.

In today's rapidly changing market, the necessary technologies are constantly evolving, while software vendors in the video domain are playing catch-up with the competition, providing little resource to invest and focusing on current integration and interoperability concerns. To further complicate matters, the quality of APIs and XML/socket interfaces to allow easy integration into the wider enterprise varies by vendor. What we have found surprising is how proprietary, unreliable or unfinished these APIs sometimes are. It is clear that several of these integration APIs - particularly those promising more exotic integration scenarios often deviate from integration standards, like $\mathrm{XML}$, or demonstrate no thought for enterprise concerns such as clustering or failover.

Going beyond the technical issues alone, there are other hurdles such as the lack of skills or project management disciplines. This is why 70 per cent of projects fail according to some studies.

\section{CHALLENGE 4 - USER- GENERATED CONTENT}

At a time of increased blogging and cheaper consumer electronics, such as digital and DV cameras, people are creating their own 
digital content like never before. The " 15 minutes of fame" axiom has never been more apt. User-generated content is typically short-form and low-quality, carrying no metadata or rights clearance. From text and images to music and video - such fodder can be valuable to marketers, enabling them to enjoy a stronger relationship with customers and vice-versa.

But what exactly are you going to do with it all? The first challenge is filtering and validating content, particularly video and audio. This can be a laborious task when the content is in different file formats. To get around the issue, you need to consider technologies that are able to validate and extract all incoming video and audio formats, codec, parameter and all metadata information and then transcode it. This removes several of the administrative tasks including informing the end-user whether their content is correctly formatted.

\section{CHALLENGE 5 - METADATA MANAGEMENT}

When sharing video around the enterprise, there is usually a need for additional data to accompany the content. Often times, the file contains insufficient information to describe the video/audio contents, the associated rights and scheduling information.

Unfortunately, in the world of metadata, the standards paradox rears its ugly head again. Unlike with images, there is no single metadata standard, and as a consequence, there are many competing ways of storing metadata - some being more structured than others.

This, then, places a burden on asset managers to catalog the content in a structured manner, either by enforcing strict metadata schemas or by parsing and reformatting a growing number of incoming metadata schemas.

\section{A SOLUTION: GETTY IMAGES MEDIA MANAGER}

These challenges highlighted the need in the market for an industrial-strength asset management solution whereby customers around the world (both big and small) could quickly set up a video library to manage and distribute their own video footage in a scalable, controlled and cost-effective manner.
As a result, Getty Images enhanced its awardwinning Media Manager service to provide an on-demand utility for companies to easily and quickly store, manage and share their video footage. Targeting marketing and media organizations around the globe, the web application solution is delivered as a "Software as a Service" (SaaS) model. This enables companies to automate the processes of uploading, transcoding and streaming digital video. The service is available in 17 languages, and customers license the service with a simple monthly subscription fee depending on the size of the asset collection and user base.

The solution integrates three technologies into the Media Management Services platform:

- VAPP (Video Asset Pipeline Processor) acts as a service-oriented, video content processing system developed by Nativ for Getty Images. It effectively offers an enterprise interface for handling all aspects of video processing including transcoding, file validation, metadata extraction, cataloging and metadata insertion. In addition, it wraps some of the finer-grained components such as FlipFactory and Mio.

- Nativ's Mio Core system was developed for cataloging, validating, filtering and repurposing video content. It enables Media Manager to identify and validate every well-known video format and codec, plus extract a huge amount of information very rapidly.

- Telestream's FlipFactory is the quick, powerful and scalable solution for transcoding media and automating video workflows. FlipFactory ensures maximum choice, where selection of specific video and audio formats and standards is concerned, and it provides a pluggable architecture for supporting future codecs. The FlipFactory application is controlled via the VAPP (Java and $\mathrm{XML}$ integration interfaces) so that transcode jobs can automatically be initiated and executed on the fly according to configurable, user-specific preferences.

The entire software infrastructure takes advantage of J2EE integration technologies and clustering systems (see Figure 1) to ensure that Media Manager can scale effortlessly with increased demand.

The video processing systems are available inline, providing content creators with an 


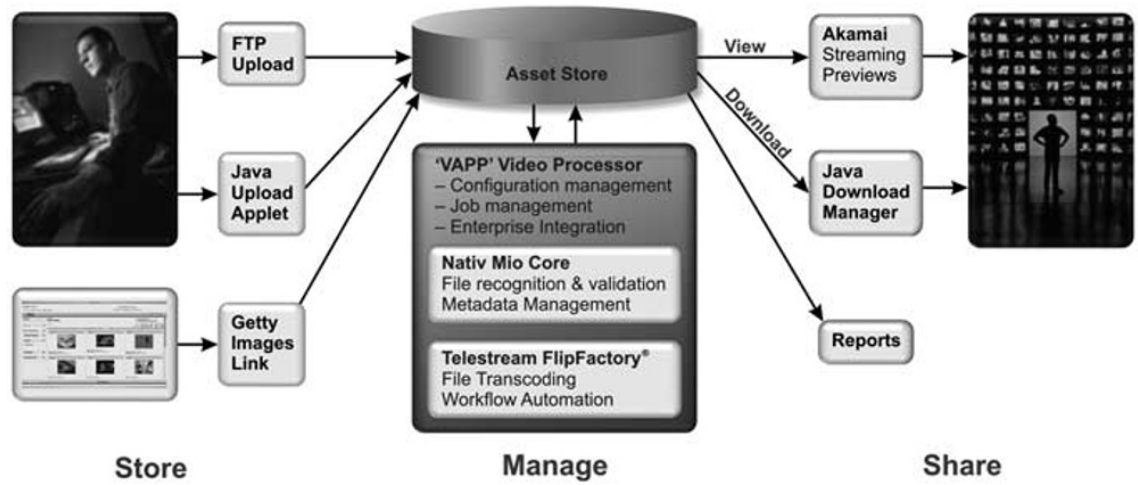

Figure 1: System diagram with video processor components

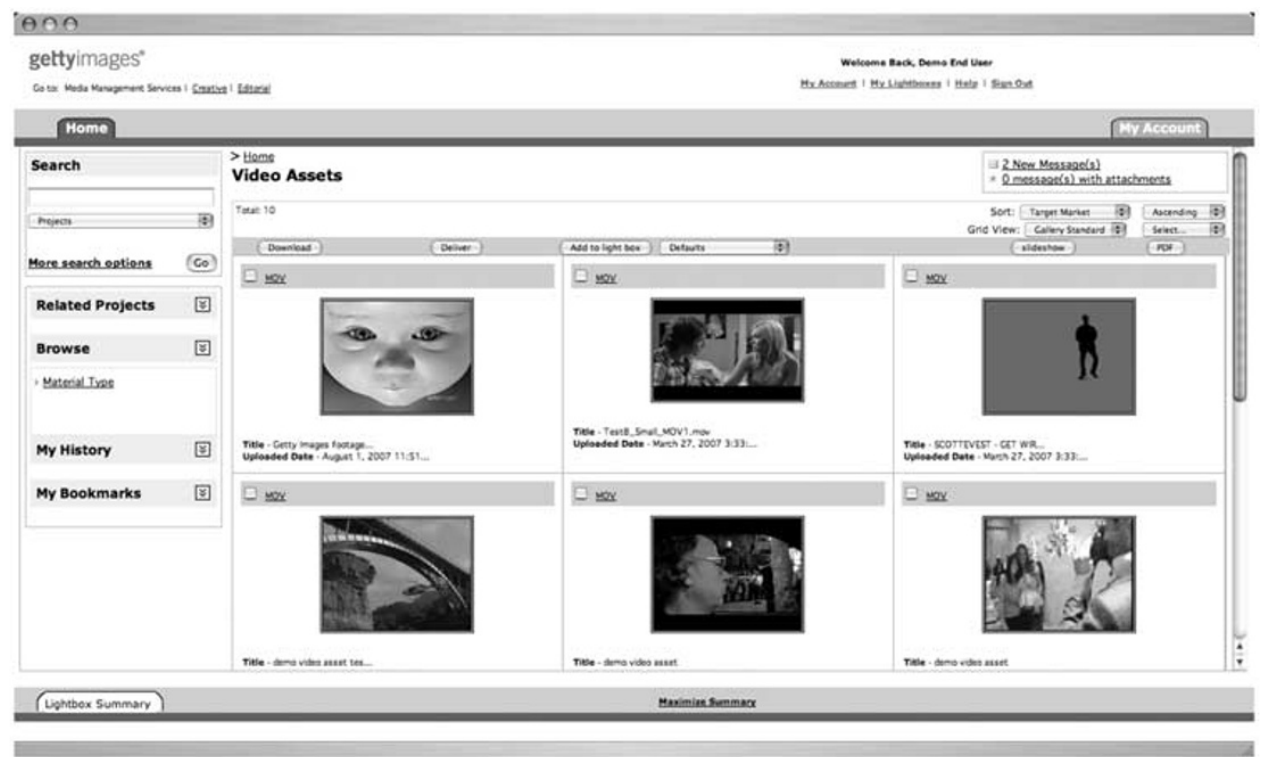

Figure 2: End user interface with video assets

automated end-to-end workflow when required. A description of the video workflow supported in the service is given below.

- When a video asset is uploaded, Media Manager uses VAPP and FlipFactory to transcode the file to a variety of video formats and codecs. There are three phases:

- To represent the asset in the user interface, a thumbnail is generated from one of the 16 key frames detected in the file (see Figure 2).

- Extracts system, structural and descriptive metadata for the video file

- System metadata (common to all files): file name, file size, etc;
- Structural metadata (common to all video): duration, frame size, etc;

- Descriptive metadata (common to some supported formats): title, description, etc.

- Generates streaming previews or new downloadable assets based on user-specific preferences setup in Media Manager.

- The original video asset and transcoded files are then stored in Media Manager. Streaming preview files are sent to Akamai.

- When users locate the video file in the Media Manager library via a search, they can view the thumbnail, play the stream in a preview window (streamed from Akamai 
servers) and download the file to their desktop - all from their web browser (see Figure 3).

The service provides a number of important benefits for customers:

- Increases collaboration via sharing of digital video with employees, partners and customers - all users need is a web browser.

- Reduces the workload on staff or production costs incurred with manual transcoding of video files.

- Reduces setup time - service can be activated in as little as 24 hours.

- Avoids investment in infrastructure (such as transformation or streaming servers) or integration projects.

- Improves security of sharing files using streaming technologies, as they are not downloaded to a user's desktop.

- Eliminates the hassles and cost of storing and shipping tapes - and the frustration of FTP.

- Increases the reliability of large file download with Java Download Manager.

\section{Conclusion}

We have covered many of the challenges that companies may face to survive in the new 360-degree, nonlinear world, and how it may push them beyond the comfort zones in order to keep up a proliferation of formats and devices. We have also suggested a potential solution. If you are interested in learning more about Getty Images Media Manager, please visit www.gettyimages.com/ mediamanager. If you are interested in strategic video consulting, please visit www.nativ.tv.

To finish, we have distilled some of the key pieces of advice we would offer to those attempting to exploit the new 360-degree world:

1. Stay away from big-bang projects.

2. Do the minimum to suit the immediate business need.

3. Be nimble and reactive.

4. Adopt a risk-sharing approach until models are proven.

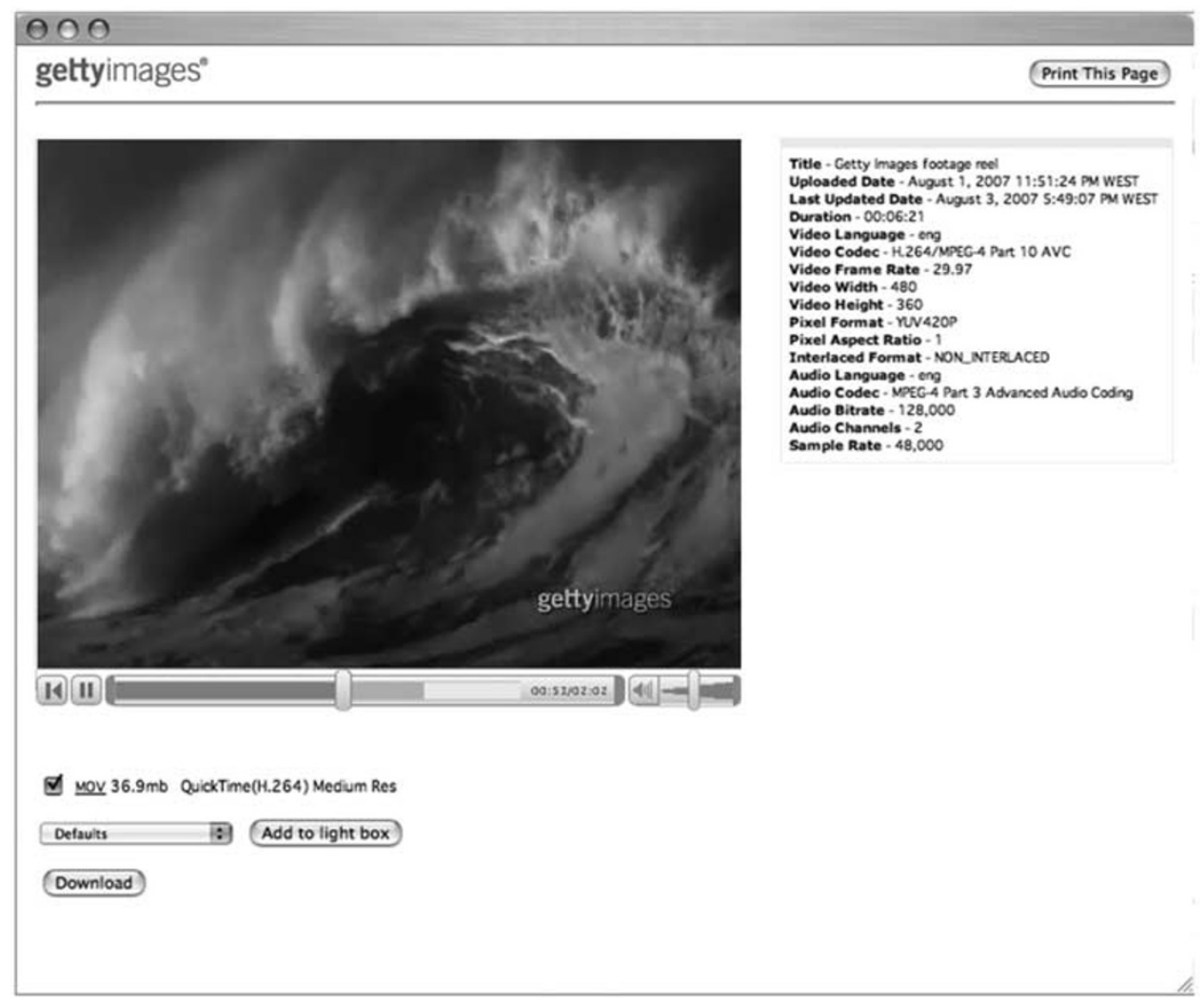

Figure 3: Preview view of streaming preview in Flash format and video metadata 
5. Rely on domain-level expertise within your own organization.

6. Do not treat product vendors as systems integrators.

7. Be cautious of "turnkey" and "out-of-thebox" promises.

8. Rely on systems integration domain specialists.
9. Apply time-tested, software engineeringbased methodologies.

10. Do not become a product development company.

11. Outsource and partner with industry experts, not generic off-shore companies.

12. Enjoy the ride. 